Pacific Journal of Mathematics

NOTE ON PROJECTIONS OF REAL ALGEBRAIC VARIETIES

A 


\title{
A NOTE ON PROJECTIONS OF REAL ALGEBRAIC VARIETIES
}

\author{
C. Andradas and J. M. Gamboa
}

\begin{abstract}
We prove that any regularly closed semialgebraic set of $R^{n}$, where $R$ is any real closed field and regularly closed means that it is the closure of its interior, is the projection under a finite map of an irreducible algebraic variety in some $R^{n+k}$. We apply this result to show that any clopen subset of the space of orders of the field of rational functions $K=R\left(X_{1}, \ldots, X_{n}\right)$ is the image of the space of orders of a finite extension of $K$.
\end{abstract}

1. Introduction. Motzkin shows in $[\mathbf{M}]$ that every semialgebraic subset of $R^{n}, R$ an arbitrary real closed field, is the projection of an algebraic set of $R^{n+1}$. However, this algebraic set is in general reducible, and we ask whether it can be found irreducible.

This turns out to be closely related to the following problem, proposed in [E-L-W]: let $K=R\left(X_{1}, \ldots, X_{n}\right), X_{1}, \ldots, X_{n}$ indeterminates, and let $X_{K}$ be the space of orders of $K$ with Harrison's topology. If $E \mid K$ is an ordered extension of $K$, let $\varepsilon_{E \mid K}$ be the restriction map between the space of orders, $\varepsilon_{E \mid K}: X_{E} \rightarrow X_{K}: P \mapsto P \cap K$. Which clopen subsets of $X_{K}$, that is, closed and open in Harrison's topology, are images of $\varepsilon_{E \mid K}$ for suitable finite extension of $K$ ?

In this note we prove that every regularly closed semialgebraic subset $S \subset R^{n}-S$ is the closure in the order topology of its inner points - is the projection of an irreducible algebraic set of $R^{n+k}$ for some $k \geq 1$. Actually we prove more: the central locus of the algebraic set, i.e., the closure of its regular points, covers the whole semialgebraic $S$. This allows us to prove that there exists an irreducible hypersurface in $R^{n+1}$ whose central locus projects onto $S$. As a consequence we prove that for every clopen subset $Y \subset X_{K}$ there is a finite extension $E$ of $K$ such that $\operatorname{im}\left(\varepsilon_{E \mid K}\right)=Y$.

2. In what follows $R$ will be a real closed field and $\pi$ will always denote the canonical projection of some $R^{n+k}$ onto the first $n$ coordinates.

Let $S$ be a semialgebraic closed subset of $R^{n}$. Then $S$ can be written in the form (cf. [C-C] $[\mathbf{R}])$ :

$$
S=\bigcup_{i=1}^{p}\left\{x \in R^{n}: f_{i 1}(x) \geq 0, \ldots, f_{i r}(x) \geq 0\right\}, \quad f_{l j} \in R\left[X_{1}, \ldots, X_{n}\right] .
$$


Now, since if $f=g \cdot h$ we have

$$
\{f \geq 0\}=\{h \geq 0, g \geq 0\} \cup\{-h \geq 0,-g \geq 0\},
$$

by decomposing each $f_{l j}$ in irreducible factors, we may assume that all of the $f_{i j}$ are irreducible. Finally, by the distributive law, we write

$$
S=\bigcap_{\left(i_{1}, \ldots, i_{p}\right) \in\{1, \ldots, r\}^{p}}\left[\left\{f_{1 i_{1}} \geq 0\right\} \cup \cdots \cup\left\{f_{p l_{p}} \geq 0\right\}\right] .
$$

For the sake of simplicity, we order the set of $p$-tuples $\left(i_{1}, \ldots, i_{p}\right)$ from 1 till $m=r^{p}$. Thus we have

$$
S=S_{1} \cap \cdots \cap S_{m}
$$

where

$$
S_{l}=\left\{f_{1 i} \geq 0\right\} \cup \cdots \cup\left\{f_{p l} \geq 0\right\}, \quad i=1, \ldots, m,
$$

and $f_{k, l}$ irreducible for all $k=1, \ldots, p ; i=1, \ldots, m$.

2.1. Proposition. Let $f_{1}, \ldots, f_{p}$ be irreducible polynomials in $R\left[X_{1}, \ldots, X_{n}\right]$. Then there exists an irreducible polynomial $F\left(T, X_{1}, \ldots, X_{n}\right)$ $\in R\left[X_{1}, \ldots, X_{n}, T\right]$ such that if $V=\left\{\underline{x} \in R^{n+1}: F(\underline{x})=0\right\}$ then

$$
\pi(V)=\left\{f_{1} \geq 0\right\} \cup \cdots \cup\left\{f_{p} \geq 0\right\} .
$$

2.2. Remark. In particular if $\{f,>0\} \neq \varnothing$ for some $j$, then $\operatorname{dim} V=$ $\operatorname{dim} S=n$ and therefore $R\left[X_{1}, \ldots, X_{n}, T\right] /(F)$ is a real domain. Thus $V$ is an irreducible hypersurface of $R^{n+1}$ which projects onto $S$.

Proof of 2.1. Set $S=\left\{f_{1} \geq 0\right\} \cup \cdots \cup\left\{f_{p} \geq 0\right\}$. The cases $S=R^{n}$, $S=\varnothing$ and $p=1$ are trivial. So, we assume $S$ proper and $p \geq 2$. Also, if for some $f_{l}$ we have $\left\{f_{i} \geq 0\right\} \subset U_{j \neq l}\left\{f_{J} \geq 0\right\}$, we just omit it, so that we may suppose the expression of $S$ irredundant in this sense. To prove the proposition we shall exhibit an irreducible polynomial $F\left(T, X_{1}, \ldots, X_{n}\right) \in$ $R\left[X_{1}, \ldots, X_{n}, T\right]$ such that the set $F=0$ projects onto $S$. Let us say a single word about how this (rather messy) polynomial comes out. We first seek an irreducible hypersurface in $R^{p+1}$ which projects over $\left\{X_{1} \geq 0\right\} \cup$ $\cdots \cup\left\{X_{p} \geq 0\right\}$. The hypersurface defined by clearing denominators in

$$
X_{p}=\frac{T^{2}\left(T^{2}-2 X_{1}\right)}{T^{2}-X_{1}}+\cdots+\frac{T^{2}\left(T^{2}-2 X_{p-1}\right)}{T^{2}-X_{p-1}}
$$


verifies this property. Thus, we substitute the $X_{i}$ 's by the $f_{i}$ 's and we check that we can modify a bit the equation above so that it keeps irreducible.

Precisely, consider the algebraic subset $V$ of $R^{n+1}$ defined by the polynomial $F\left(T, X_{1}, \ldots, X_{n}\right)$ obtained by clearing denominators in the equation

$$
f_{p}=\frac{T^{2}\left(T^{2}-\lambda_{1} f_{1}\right)}{T^{2}-\lambda_{2} f_{1}}+\sum_{i=2}^{p-1} \frac{T^{2}\left(T^{2}-2 f_{i}\right)}{\left(T^{2}-f_{i}\right)}
$$

where $\lambda_{1}, \lambda_{2} \in R, 0<\lambda_{2}<\lambda_{1}$. That is, if we set:

$$
\begin{aligned}
& Q(T, \underline{X})=\prod_{i=2}^{p-1}\left(T^{2}-f_{i}\right), \\
& Q_{t}(T, \underline{X})=Q(T, \underline{X}) /\left(T^{2}-f_{i}\right) \quad(i=2, \ldots, p-1)
\end{aligned}
$$

then

$$
\begin{aligned}
F(T, \underline{X})= & Q f_{p}\left(T^{2}-\lambda_{2} f_{1}\right)-Q T^{2}\left(T^{2}-\lambda_{1} f_{1}\right) \\
& -\left(T^{2}-\lambda_{2} f_{1}\right) \sum_{i=2}^{p-1} T^{2}\left(T^{2}-2 f_{l}\right) Q_{l} .
\end{aligned}
$$

We claim that $\pi(V)=S$. Indeed, let $a \in S$. If $f_{i}(q)=0$ for some $i=1, \ldots, p-1$, then it is immediate that the point $(a, 0) \in V$. So we restrict ourselves to the case $f_{i}(a) \neq 0$ for all $i=1, \ldots, p-1$. Now notice that the graph of the functions (in the plane)

$$
Y=\frac{T^{2}\left(T^{2}-2 f_{i}(a)\right)}{T^{2}-f_{i}(a)} \quad(i=2, \ldots, p-1)
$$

as well as

$$
Y=\frac{T^{2}\left(T^{2}-\lambda_{1} f_{1}(a)\right)}{T^{2}-\lambda_{2} f_{1}(a)} \quad\left(0<\lambda_{2}<\lambda_{1}\right)
$$

look like Figure 1 if $f_{i}(a)<0$ (resp. $f_{1}(a)<0$ ) and like Figure 2 if $f_{i}(a)>0$ (resp. $f_{1}(a)>0$, where we have to change $\sqrt{2 f_{i}(a)}$ and $\sqrt{f_{i}(a)}$ by $\sqrt{\lambda_{1} f_{1}(a)}$ and $\left.\sqrt{\lambda_{2} f_{1}(a)}\right)$.

Thus, the range of the function

$$
Y=\frac{T^{2}\left(T^{2}-\lambda_{1} f_{1}(a)\right)}{T^{2}-\lambda_{2} f_{1}(a)}+\sum_{i=2}^{p-1} \frac{T^{2}\left(T^{2}-2 f_{l}(a)\right)}{T^{2}-f_{i}(a)}
$$

is either the whole line $R$ if $f_{i}(a)>0$ for some $i=1, \ldots, p-1$, or $Y \geq 0$ if $f_{l}(a)<0$ for all $i=1, \ldots, p-1$. Since in this case we have $f_{p}(a) \geq 0$ 
(by the very definition of $S$ ), it is clear that for any $a \in S$ there exists $t \in R$ such that $\left(t, f_{p}(a)\right)$ verifies (2.1.2). Obviously this means that the point $(a, t) \in V$ and so $a \in \pi(V)$. This shows $S \subset \pi(V)$.

The converse is immediate, for, if $a \notin S$ then $f_{i}(a)<0$ for all $i=1, \ldots, p$. But, by the definition of $V,(a, t) \in V$ and $f_{1}(a)<$ $0, \ldots, f_{p-1}(a)<0$, imply $f_{p}(a) \geq 0$, and so $a \notin \pi(V)$ if $a \notin S$.

Finally, the following Lemma 2.3 shows that there exist $\lambda_{1}, \lambda_{2}$, $0<\lambda_{2}<\lambda_{1}$, such that $F\left(T, X_{1}, \ldots, X_{n}\right)$ is irreducible, what concludes the proof of 2.1 .

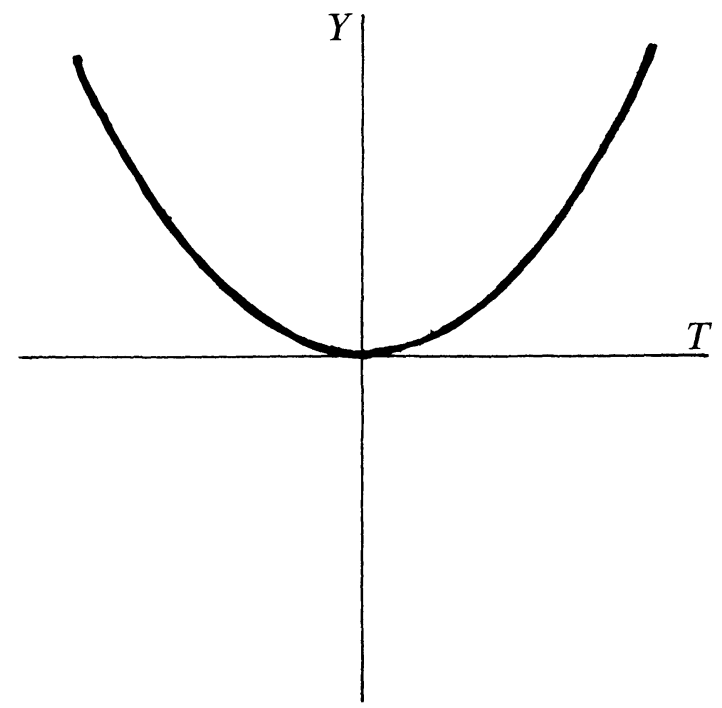

FIGURE 1

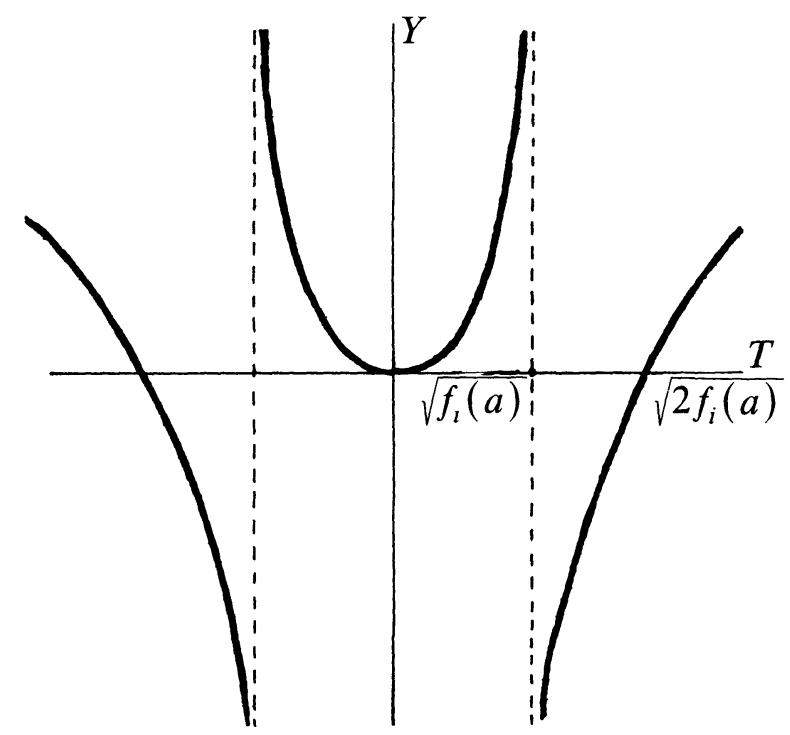

FIGURE 2 
2.3. Lemma. Let $f_{1}, \ldots, f_{p}, p \geq 2$ be irreducible polynomials in $R\left[X_{1}, \ldots, X_{n}\right]$, such that $S=\left\{f_{1} \geq 0\right\} \cup \cdots \cup\left\{f_{p} \geq 0\right\}$ is irredundant (i.e. $\left\{f_{l} \geq 0\right\} \not \subset \cup_{j \neq l}\left\{f_{j} \geq 0\right\}$ for all $\left.i\right)$ and $S$ is neither $R^{n}$ nor empty. Then there exist $\lambda_{1}, \lambda_{2} \in R, 0<\lambda_{2}<\lambda_{1}$, such that the polynomial $F(T, \underline{X})$ defined in (2.1.1) is irreducible.

Proof. The result is a consequence of Bertini's theorem ${ }^{1}$. To see this, we write $F(T, \underline{X})$ in the form

$$
F(T, \underline{X})=P_{0}+\lambda_{1} P_{1}+\lambda_{2} P_{2}
$$

where

$$
\begin{aligned}
& P_{0}=Q f_{p} T^{2}-Q T^{4}-T^{4} \sum_{i=2}^{p-1}\left(T^{2}-2 f_{l}\right) Q_{i} \\
& P_{1}=Q f_{1} T^{2} \\
& P_{2}=f_{1} T^{2} \sum_{i=2}^{p-1}\left(T^{2}-2 f_{i}\right) Q_{l}-Q f_{1} f_{p} .
\end{aligned}
$$

Now, if $C=R(\sqrt{-1})$, set

$$
Z=\left\{(\underline{x}, t) \in C^{n+1}: P_{0}(\underline{x}, t)=P_{1}(\underline{x}, t)=P_{2}(\underline{x}, t)=0\right\}
$$

and consider $\phi: C^{n+1} \backslash Z \rightarrow \mathbf{P}_{2}(C)$ defined by

$$
\phi\left(x_{1}, \ldots, x_{n}, t\right)=\left(P_{0}(\underline{x}, t), P_{1}(\underline{x}, t), P_{2}(\underline{x}, t)\right) .
$$

Let $\Lambda$ be the set of points $\left(\lambda_{1}, \lambda_{2}\right) \in C^{2}$ such that $\left\{P_{0}+\lambda_{1} P_{1}+\right.$ $\left.\lambda_{2} P_{2}=0\right\}$ is irreducible and non-singular (as a subvariety of $C^{n+1} \backslash Z$ ). Then Bertini's theorem (cf. [H], pag. 275) assures that $\Lambda$ contains a Zariski open subset of $C^{2}$ provided that

(a) $\operatorname{dim}(\operatorname{im} \phi)=2$.

Furthermore, if

(b) $P_{0}, P_{1}$ and $P_{2}$ are relatively prime, then $Z$ has codimension $\geq 2$, hence $\left\{P_{0}+\lambda_{1} P_{1}+\lambda_{2} P_{2}=0\right\}$ is irreducible in $C^{n+1}$.

Thus since open intervals of $R$ are Zariski-dense in $C$, the result follows at once if we prove (a) and (b). Let us begin with the second:

(b) Assume that $h(\underline{X}, T)$ is an irreducible common factor of $P_{0}, P_{1}$ and $P_{2}$.

Then $h \mid P_{1}$ and so, we have $h=T, h=f_{1}$ or $h \mid Q$. Since $P_{2}(0, \underline{X})=$ $(-1)^{p-1} \prod_{i=1}^{p} f_{l} \neq 0$, it follows that $T+P_{2}$.

\footnotetext{
${ }^{1}$ We want to thank Professor J. P. Serre who called our attention to Bertini's theorem in order to prove 2.3 .
} 
Now, suppose $h=f_{1}$. Since $h \mid P_{0}$, we have

$$
f_{1} \mid\left(Q f_{p}-T^{2} Q-T^{2} \sum_{i=2}^{p-1}\left(T^{2}-2 f_{i}\right) Q_{l}\right) \text {. }
$$

In particular, setting $T=0, f_{1} \mid\left((-1)^{p-2} \prod_{l=2}^{p} f_{i}\right)$, which implies, since $f_{1}$ is irreducible, that there exist $a \in R$ and $j \in\{2, \ldots, p\}$ such that $f_{1}=a f_{j}$. But $a>0$ means $\left\{f_{1} \geq 0\right\}=\left\{f_{j} \geq 0\right\}$, and $S$ would not be irredundant, while $a<0$ implies $S=R^{n}$. Therefore $h \neq f_{1}$.

Finally, suppose $h \mid Q$. Then, we have $h=T^{2}-f_{J}$ for some $j=2, \ldots$, $p-1$. Since $h \mid P_{0}$, we deduce

$$
h \mid \sum_{i=2}^{p-1} Q_{i} \cdot\left(T^{2}-2 f_{i}\right) .
$$

But $h$ divides $Q_{i}$ for all $i \neq j$. Thus $h \mid Q_{j}\left(T^{2}-2 f_{j}\right)$ which is absurd. This ends the proof of (b).

(a) It is enough to check that there is no homogeneous polynomial $H\left(Y_{0}, Y_{1}, Y_{2}\right) \in C\left[Y_{0}, Y_{1}, Y_{2}\right]-\{0\}$ such that $H\left(P_{0}, P_{1}, P_{2}\right) \equiv 0$. Suppose the opposite and assume that $H$ is of degree $d$. Then

$$
H\left(Y_{0}, Y_{1}, Y_{2}\right)=\sum_{a+b+c=d} \alpha_{a b c} Y_{0}^{a} Y_{1}^{b} Y_{2}^{c} .
$$

We shall work on the lowest degree in $T$ of the monomials $P_{0}^{a} P_{1}^{b} P_{2}^{c}$. From (2.3.1) we get

$$
\begin{aligned}
P_{0}^{a} P_{1}^{b} P_{2}^{c}= & \left(\prod_{i=2}^{p-1}\left(-f_{i}\right)\right)^{d}(-1)^{c} f_{1}^{b+c} f_{p}^{a+c} T^{2(a+b)} \\
& +T^{2(a+b)+1} G(X, T)
\end{aligned}
$$

(where in the case $p=2$ the first product is taken to be 1).

We will prove that $\alpha_{a b c}=0$ for all $a, b, c$. Set $h=a+b$. We work by induction on $h$.

If $h=0$, then $a=b=0$ and we have to prove that $\alpha_{0,0, d}=0$. But the independent term of $H\left(P_{0}, P_{1}, P_{2}\right)$ is $\alpha_{0,0, d} \cdot\left(\Pi_{l=1}^{p} f_{i}\right)^{d}$. Then $\alpha_{0,0, d}=0$. Suppose $\alpha_{a^{\prime} b^{\prime} c^{\prime}}=0$ whenever $a^{\prime}+b^{\prime}<h$. Then

$$
H\left(P_{0}, P_{1}, P_{2}\right)=\sum_{\substack{a+b+c=d \\ a+b \geq h}} \alpha_{a b c} P_{0}^{a} P_{1}^{b} P_{2}^{c}=T^{2 h} M(T, \underline{X})
$$

Since we have seen that $P_{0}^{a} P_{1}^{b} P_{2}^{c}=T^{2(a+b)} \cdot R(T, \underline{X})$, the term of degree $2 h$ in $H\left(P_{0}, P_{1}, P_{2}\right)$ comes from those $a, b, c$ such that $a+b=h$ and its 
coefficient is, after (2.3.3),

$$
\sum_{\substack{a+b+c=d \\ a+b=h}} \alpha_{a b c}(-1)^{d}\left(\prod_{l=2}^{p-1} f_{l}\right)^{d}(-1)^{c} f_{1}^{b+c} f_{p}^{a+c} .
$$

Thus, we obtain

$$
\sum_{i=0}^{h} \alpha_{l, h-i, d-h} f_{1}^{d-\imath} f_{p}^{d-h+\imath}=0
$$

which implies

$$
\sum_{l=0}^{h} \alpha_{l, h-l, d-h}\left(f_{p} / f_{1}\right)^{i}=0 .
$$

But, if $\alpha_{i, h-i, d-h} \neq 0$ for some $i$, this means that $f_{p} / f_{1}$ is algebraic over $C$, hence $f_{p}=\lambda f_{1}, \lambda \in C$. Moreover, since $f_{1}, f_{p} \in R\left[X_{1}, \ldots, X_{n}\right]$, we know that $\lambda \in R$. Repeating a foregoing argument, $\lambda>0$ means $\left\{f_{1} \geq 0\right\}=$ $\left\{f_{p} \geq 0\right\}$ and $\lambda<0$ means $S=R^{n}$. Since both cases have been eliminated it follows $\alpha_{a b c}=0$ whenever $a+b=0$ and the proof of the lemma is complete.

3. The main result. From now on, given an algebraic set $V, V_{c}$ will denote the set of central points of $V$, that is the closure of the regular points of $V$. We start with:

3.1. Definition. A semialgebraic subset $S$ of $R^{n}$ is regularly closed if $S$ is the closure of its inner points.

We are now ready to prove the following:

3.2. THEOREM. Let $S \subset R^{n}$ be a closed semialgebraic set of dimension $n$. There exists a positive integer $m$ and an irreducible $n$-dimensional algebraic set $V \subset R^{n+m}$ such that

(1) $\pi: V \rightarrow R^{n}$ is finite,

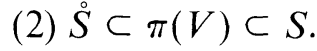

Moreover, if $S$ is regularly closed then $\pi\left(V_{c}\right)=\pi(V)=S$.

Proof. We may assume $S$ written in the form (2.0.1), i.e.

$$
S=S_{1} \cap \cdots \cap S_{m}, \quad \text { with } S_{l}=\left\{f_{1 l} \geq 0\right\} \cup \cdots \cup\left\{f_{p l} \geq 0\right\}
$$


and $f_{k i} \in R\left[X_{1}, \ldots, X_{n}\right]$ irreducible for every $(i, k) \in\{1, \ldots, m\} \times$ $\{1, \ldots, p\}$. We will find $V \subset R^{n+m}$. To do that we work by induction on $m$.

For $m=1$, let $V \subset R^{n+1}$ be the hypersurface $F(T, \underline{X})=0$ of Proposition 2.1 if $p>1$ and $T^{2}-f_{1}=0$ if $p=1$. Notice that the leading coefficient of $F(T, \underline{X})$ as polynomial in $T$ is $1-p$ (see 2.1.1) and consequently $\pi: V \rightarrow R^{n}$ is finite. Since $\pi(V)=S$ condition (2) is trivially satisfied.

Assume now that there exists an irreducible algebraic set $W^{\prime} \subset$ $R^{n+m-1}$ of dimension $n$ verifying:

$$
\begin{aligned}
& \text { (i) } \pi: W^{\prime} \rightarrow R^{n} \text { is finite } \\
& \text { (ii) } \dot{S}^{\prime} \subset \pi\left(W^{\prime}\right) \subset S^{\prime},
\end{aligned}
$$

where $S^{\prime}=S_{1} \cap \cdots \cap S_{m-1}$ (which has, of course, dimension $n$ ).

Let $\mathscr{J}\left(W^{\prime}\right) \subset R\left[X_{1}, \ldots, X_{n}, T_{1}, \ldots, T_{m-1}\right]$ be the ideal of polynomials vanishing on $W^{\prime}$ and consider the variety $W \subset R^{n+m}$ defined by $\mathscr{J}\left(W^{\prime}\right)$. $R\left[X_{1}, \ldots, X_{n}, T_{1}, \ldots, T_{m-1}, T\right]$, where $T$ is a new variable. Obviously $W$ is irreducible and verifies the condition (ii) of (3.2.1).

Now let $F(T, \underline{X})=P_{0}+\lambda_{1} P_{1}+\lambda_{2} P_{2} \in R\left[X_{1}, \ldots, X_{n}, T\right]$ be the polynomial defined in (2.1.1) such that for any $\lambda_{1}, \lambda_{2} \in R, 0<\lambda_{2}<\lambda_{1}$, the set $V_{m}^{\prime}$ of zeros of $F$ (in $R^{n+1}$ ) projects onto $S_{m}$. Let $V_{m}$ be the algebraic set of $R^{n+m}$ defined by $F(T, \underline{X})$ considered as a polynomial in $R\left[X_{1}, \ldots, X_{n}, T_{1}, \ldots, T_{m-1}, T\right]$. We have

$$
\stackrel{\circ}{S} \subset S_{m} \cap \dot{S}^{\prime} \subset \pi\left(V_{m} \cap W\right) \subset S .
$$

Set $Z=\left\{\left(\underline{x}, t_{1}, \ldots, t_{m-1}, t\right) \in R^{n+m}: \quad P_{0}(\underline{x}, t)=P_{1}(\underline{x}, t)=P_{2}(\underline{x}, t)\right.$ $=0\}$. Since $P_{0}, P_{1}, P_{2}$ have no common factors (see proof of 2.3), it is $\operatorname{codim}(\pi(Z)) \geq 1$. Let $H=\operatorname{Sing}(W) \cup(Z \cap W)$. Then $\operatorname{codim}(\pi(H)) \geq$ 1 , since by induction hypothesis $\operatorname{dim} W^{\prime}=n$. Let $C=R(\sqrt{-1})$ be the algebraic closure of $R$ and consider $\phi: W \backslash H \rightarrow \mathbf{P}_{2}(C)$ defined by

$$
\phi\left(\underline{x}, t_{1}, \ldots, t_{m-1}, t\right)=\left(P_{0}(\underline{x}, t), P_{1}(\underline{x}, t), P_{2}(\underline{x}, t)\right) .
$$

Since $W \backslash H$ is non-singular, Bertini's theorem applies assuring that the set of points $\left(\lambda_{1}, \lambda_{2}\right) \in C^{2}$ such that

$$
(W \backslash H) \cap\left\{\left(\underline{x}, t_{1}, \ldots, t_{m-1}, t\right): P_{0}(\underline{x}, t)+\lambda_{1} P_{1}(\underline{x}, t)+\lambda_{2} P_{2}(\underline{x}, t)=0\right\}
$$

is irreducible and non-singular (as a subvariety of $W \backslash H$ ) contains a Zariski open subset of $C^{2}$, provided that $\operatorname{dim}(\operatorname{im} \phi)=2$.

Since $\pi(W)$ has non-empty interior, to prove that $\operatorname{dim}(\operatorname{im} \phi)=2$ it is enough to show that $P_{0}, P_{1}$ and $P_{2}$ do not verify any homogeneous 
polynomial. But this was shown in the proof of Lemma 2.3. Therefore there exist $\lambda_{1}, \lambda_{2} \in R, 0<\lambda_{2}<\lambda_{1}$, such that $V_{m} \cap(W \backslash H)$ is irreducible and nonsingular (in $W \backslash H$ ). Let $V$ be the irreducible component of $V_{m} \cap W$ which coincides with $V_{m} \cap(W \backslash H)$ on $W \backslash H$. Thus $\operatorname{dim} V \leq n$ and from $\operatorname{codim}(\pi(H)) \geq 1$ it follows $\operatorname{dim} V=\operatorname{dim}\left(W \cap V_{m}\right)=n$.

Since the morphisms $\pi: W^{\prime} \rightarrow R^{n}$ and $\pi: V_{m} \rightarrow R^{n}$ are finite so is $\pi:$ $V_{m} \cap W \rightarrow R^{n}$, which implies the finiteness of $\pi: V \rightarrow R^{n}$. Whence $\pi(V)$ is closed in $R^{n}$. Obviously $\pi(V) \subset S$. Let us see that $\stackrel{S}{S} \subset \pi(V)$. Let $x \in \dot{S}$ and let $U \subset S \stackrel{S}{ }$ be a strong open neighborhood of $x$. Since $\operatorname{codim}(\pi(H)) \geq$ 1 , we deduce that $U \cap(\dot{S} \backslash \pi(H)) \neq \varnothing$. Take $y \in U \cap(\stackrel{\circ}{S} \backslash \pi(H))$. Then $y \in \pi\left(W^{\prime}\right) \cap \pi\left(V_{m}^{\prime}\right)$. Pick $\left(t_{1}, \ldots, t_{n-1}\right)=t^{\prime} \in R^{m-1}$ and $t \in R$ such that $\left(y, t^{\prime}\right) \in W^{\prime}$ and $(y, t) \in V_{m}^{\prime}$. We have $\left(y, t^{\prime}, t\right) \in\left(W \cap V_{m}\right) \backslash H \subset V$. Hence $U \cap \pi(V) \neq \varnothing$ and since $\pi(V)$ is closed we conclude that $\dot{S} \subset$ $\pi(V)$, what proves the first part of the theorem.

Finally, assume that $S$ is regularly closed. First of all notice that, since $\pi$ is finite, $\pi\left(V_{c}\right)$ is a closed semialgebraic subset of $R^{n}$ (see [B], page 170). From $\stackrel{\circ}{S} \subset \pi(V)$ it follows that $\stackrel{\circ}{S} \subset \pi\left(V_{c}\right)$. For let $x \in \stackrel{\circ}{S} \backslash \pi\left(V_{c}\right)$ and let $U \subset S$ be a strong open neighborhood of $x$ such that $U \cap \pi\left(V_{c}\right)=\varnothing$. Thus $U \subset \pi\left(V \backslash V_{c}\right)$; but $\operatorname{dim} \pi\left(V \backslash V_{c}\right)<n=\operatorname{dim} U$, contradiction. Therefore we have $\stackrel{S}{S} \subset \pi\left(V_{c}\right) \subset \pi(V) \subset S$. Taking into account once more that both $\pi\left(V_{c}\right)$ and $\pi(V)$ are closed and that $S$ is regularly closed, it follows at once by taking closures that $\pi\left(V_{c}\right)=\pi(V)=S$ and Theorem 3.1 is complete.

3.3. Corollary. Let $S \subset R^{n}$ be a regularly closed semialgebraic set. Then there exists an irreducible algebraic hypersurface $\tilde{V} \subset R^{n+1}$ such that $\pi\left(\tilde{V}_{c}\right)=S$.

Proof. Let $V \subset R^{n+m}$ be the irreducible algebraic variety constructed in 3.2 , and let $C=R\left[X_{1}, \ldots, X_{n}, x_{n+1}, \ldots, x_{n+m}\right]$ be its coordinate ring. Then $\pi\left(V_{c}\right)=\pi(V)=S$ and $C$ is integral over $A=R\left[X_{1}, \ldots, X_{n}\right]$. Let $t=\lambda_{1} X_{n+1}+\cdots+\lambda_{m} X_{n+m}, \lambda_{l} \in R$, be a primitive element of $R(V)$ over $R\left(X_{1}, \ldots, X_{n}\right)$ and let $\tilde{V}$ be the hypersurface of $R^{n+1}$ with coordinate ring $B=R\left[X_{1}, \ldots, X_{n}, t\right]$. Then we have the following diagram,

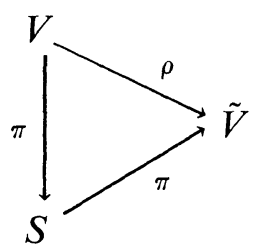


where all the morphisms are finite, $\pi$ represents the projection on the first $n$ coordinates, and $\rho$ induces a birational isomorphism. Therefore $\rho\left(V_{c}\right)=$ $\tilde{V}_{c}$ (see [D-R], 2.9) and we get $\pi\left(\tilde{V}_{c}\right)=S$.

3.4. Remark. We still do not know whether a regularly closed semialgebraic subset of $R^{n}$ is the projection of an irreducible hypersurface of $R^{n+1}$. In case the answer is negative, is there a bound of the integer $m$ which does not depend on $S$ (i.e. an universal bound for all regularly closed semialgebraic subsets of $\left.R^{n}\right)$ ?

4. Application to Harrison's topology. Throughout this section $K=$ $R\left(X_{1}, \ldots, X_{n}\right)$ will be a pure transcendental extension of $R$ of degree $n$, and $X(K)$ will denote its space of orders. If $E$ is a formally real extension of $K$, we will denote by $\varepsilon_{E \mid K}$ the induced morphism between $X(E)$ and $X(K)$, namely

$$
\varepsilon_{E \mid K}: X(E) \rightarrow X(K): P \mapsto P \cap K .
$$

A clopen subset $Y$ of $X(K)$ is a subset which is open and closed in the Harrison's topology of $X(K)$, i.e. the topology whose basis consists of the sets:

$$
H\left(f_{1}, \ldots, f_{r}\right)=\left\{P \in X(K): f_{1} \in P, \ldots, f_{r} \in P\right\},
$$

$f_{l} \in R\left[X_{1}, \ldots, X_{n}\right]$ for all $i$.

Since $X(K)$ with Harrison's topology is compact $([\mathbf{P}])$, every clopen set $Y$ can be written as a finite union of open basic sets:

$$
Y=H_{1} \cup \cdots \cup H_{p}, \quad \text { where } H_{i}=H\left(f_{1 l}, \ldots, f_{r l}\right) \text {. }
$$

Theorem 3.2 will be used to prove the following:

4.1. TheOREM. Let $Y$ be any clopen set of $X(K)$. Then there exists a finite extension $E$ of $K$ such that $Y=\operatorname{im} \varepsilon_{E \mid K}$.

Proof. Let $Y=H_{1} \cup \cdots \cup H_{p}, \quad H_{i}=H\left(f_{1 l}, \ldots, f_{r i}\right), \quad f_{k i} \in$ $R\left[X_{1}, \ldots, X_{n}\right]$ for all $(k, i) \in\{1, \ldots, r\} \times\{1, \ldots, p\}$. Define the semialgebraic associated to $Y$ by

$$
\hat{Y}=\hat{H_{1}} \cup \cdots \cup \hat{H_{p}}
$$

where $H_{i}^{\hat{i}}=\left\{\underline{x} \in R^{n}: f_{1 l}(\underline{x})>0, \ldots, f_{r_{l}}(\underline{x})>0\right\}$. In [D-R] it is shown that the correspondence $Y \rightarrow Y^{\wedge}$ verifies that $Y_{1}=Y_{2}$ if and only if $\overline{Y_{1}^{\wedge}}$ $=\overline{Y_{2}}$, where $\overline{Y^{\wedge}}$ denotes the closure of $Y^{\wedge}$ in the strong topology of $R^{n}$. 
Since $Y^{\wedge}$ is open, $\overline{Y^{\wedge}}$ is a regularly closed semialgebraic subset of $R^{n}$. Then 2.5 applies producing an $n$-dimensional irreducible algebraic set $V \subset R^{n+m}$ such that $\pi(V)=\pi\left(V_{c}\right)=\overline{Y^{\wedge}}$. In particular, $\overline{\pi\left(V_{c}\right)}=\overline{Y^{\wedge}}$. Since $\operatorname{dim} V=n$, the function field $E$ of $V$ is a finite extension of $K$ and $R\left[X_{1}, \ldots, X_{n}\right] \rightarrow$ $R[V]$ is integral since $\pi: V \rightarrow R^{n}$ is finite.

It follows immediately from [D-R] (Prop. 2.7) that im $\varepsilon_{E \mid K}=Y$.

4.2. REMARK. In $[\mathbf{E}-\mathbf{L}-\mathbf{W}]$ is suggested that the characterization of those clopen subsets of the space of orders $X_{K}$ of a field $K$ which are the image of $\varepsilon_{E \mid K}$ for some finite extension $E \mid K$ could depend on topological properties of $\varepsilon$ for finite extensions. However, since there are examples ([E-L-W]) of clopen sets which are not $\operatorname{im}\left(\varepsilon_{E \mid K}\right)$ for any $E$, and after Theorem 4.1, it follows that such a characterization is not intrinsic to $\varepsilon$ but depends on the base field $K$.

Acknowledgment. The authors wish to thank the referee who pointed out several mistakes in the original version of the paper.

\section{REFERENCES}

[B] G. W. Brumfiel, Partially ordered fields and semialgebraic geometry, London Math. Soc. Lect. Notes, 37 (1979).

[C-C] M. Coste and M. F. Roy, La topologie du spectre réel, Contemporary Math., 8 (1982), 27-59.

[D-R] D. W. Dubois and T. Recio, Order extensions and real algebraic geometric, Contemporary Math., 8 (1982), 265-288.

[E-L-W] R. Elman, T. Y. Lam and A. Wadsworth, Orderings under field extensions, J. Reine Ang. Math., 306 (1979), 7-27.

[H] R. Hartshorne, Algebraic Geometry, G.T.M. no. 52, Springer Verlag, (1977).

[M] T. S. Motzkin, The Real Solution Set of a System of Algebraic Inequalities, Inequalities II, Academic Press (1970).

[P] A. Prestel, Lectures on Formally Real Fields, I.M.P.A. no. 25, (1975).

[R] T. Recio, Una descomposición de un conjunto semialgebraico, Actas V Congreso de Matemáticas de expresión Latina. Mallorca, Spain, (1977).

Received January 20, 1983.

DPto. De Algebra y Fundamentos

FACUlTAd DE CC. MATEMÁticas

UNIVERSIDAD COMPLUTENSE

MADRID 3, SPAIN 



\title{
PACIFIC JOURNAL OF MATHEMATICS \\ EDITORS
}

\author{
DoNALD BABBITT (Managing Editor) \\ University of California \\ Los Angeles, CA 90024 \\ J. DugunduI \\ University of Southern California \\ Los Angeles, CA 90089-1113 \\ R. FINN \\ Stanford University \\ Stanford, CA 94305 \\ HERMANN FlasChKa \\ University of Arizona \\ Tucson, AZ 85721
}

C. C. Moore

University of California

Berkeley, CA 94720

ARTHUR OGUS

University of California

Berkeley, CA 94720

Hugo Rossi

University of Utah

Salt Lake City, UT 84112

H. SAMELSON

Stanford University

Stanford, CA 94305

ASSOCIATE EDITORS
R. ARENS
E. F. BECKENBACH
B. H. NeUMANN
F. WOLF
K. YoSHIDA (1906-1982)

\section{SUPPORTING INSTITUTIONS}

UNIVERSITY OF ARIZONA
UNIVERSITY OF BRITISH COLUMBIA
CALIFORNIA INSTITUTE OF TECHNOLOGY
UNIVERSITY OF CALIFORNIA
MONTANA STATE UNIVERSITY
UNIVERSITY OF NEVADA, RENO
NEW MEXICO STATE UNIVERSITY
OREGON STATE UNIVERSITY
UNIVERSITY OF ARIZONA
CALIFORNIA INSTITUTE OF TECHNOLOGY
UNIVERSITY OF CALIFORNIA
UNIVERSITY OF NEVADA, RENO
OREGON STATE UNIVERSITY
UNIVERSITY OF OREGON
UNIVERSITY OF SOUTHERN CALIFORNIA
STANFORD UNIVERSITY
UNIVERSITY OF HAWAII
UNIVERSITY OF TOKYO
UNIVERSITY OF UTAH
WASHINGTON STATE UNIVERSITY
UNIVERSITY OF WASHINGTON 


\section{Pacific Journal of Mathematics}

Vol. 115, No. $1 \quad$ September, 1984

Carlos Andradas Heranz and José Manuel Gamboa Mutuberría, A note on projections of real algebraic varieties $\ldots \ldots \ldots \ldots \ldots \ldots \ldots \ldots \ldots \ldots$

Jürgen Appell and Maria Patrizia Pera, Noncompactness principles in nonlinear operator approximation theory $\ldots \ldots \ldots \ldots \ldots \ldots \ldots \ldots \ldots \ldots$

Timothy John Carlson, Extending Lebesgue measure by infinitely many

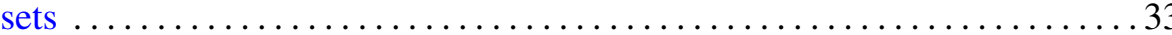

Donald S. Coram and Paul Frazier Duvall, Jr., Non-cell-like

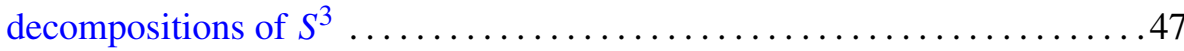

Edward Norman Dancer, Order intervals of selfadjoint linear operators and

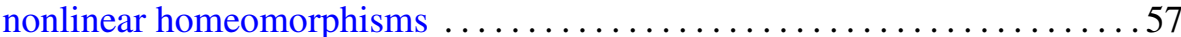

Ralph Jay De Laubenfels, Well-behaved derivations on $C[0,1] \ldots \ldots \ldots 73$

D. Feyel and A. de La Pradelle, Sur certaines extensions du théorème

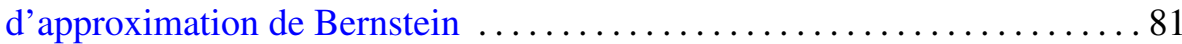

Colin C. Graham and Bertram Manuel Schreiber, Bimeasure algebras on

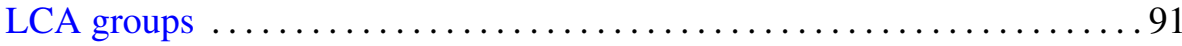

Richard Howard Hudson, Class numbers of imaginary cyclic quartic fields

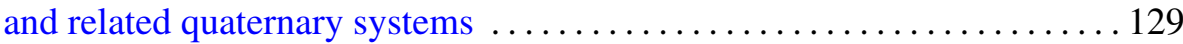

Carl Groos Jockusch, Jr. and Iraj Kalantari, Recursively enumerable sets and van der Waerden's theorem on arithmetic progressions . . . . . . . . 143

J. F. McClendon, On noncontractible valued multifunctions ........... 155 Akihiko Miyachi, Weak factorization of distributions in $H^{p}$ spaces $\ldots \ldots \ldots 165$ Ezzat S. Noussair and Charles Andrew Swanson, Global positive solutions of semilinear elliptic problems

Jon Christopher Snader, Strongly analytic subspaces and strongly

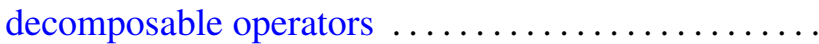

Boguslaw Tomaszewski, A construction of inner maps preserving the Haar measure on spheres

Akihito Uchiyama, The Fefferman-Stein decomposition of smooth functions and its application to $H^{p}\left(\mathbf{R}^{n}\right) \ldots \ldots \ldots$ 